\title{
G-CSF, rt-PA and combination therapy after experimental thromboembolic stroke
}

\author{
Rainer Kollmar*1, Nils Henninger2 ${ }^{2}$, Christian Urbanek ${ }^{3}$ and Stefan Schwab
}

\begin{abstract}
Background: Granulocyte Colony-Stimulating Factor (G-CSF) has remarkable neuroprotective properties. Due to its proven safety profile, G-CSF is currently used in clinical stroke trials. As neuroprotectants are considered to be more effective in the early phase of cerebral ischemia and during reperfusion, G-CSF should to be tested in combination with thrombolysis. Therefore, combination therapy was investigated in an experimental model of thromboembolic stroke.

Methods: Male Wistar rats ( $n=72$ ) were subjected to a model of thromboembolic occlusion (TE) of the middle cerebral artery. Different groups ( $n=12$ each) treated by recombinant tissue-plasminogen activator (rt-PA) or/and GCSF: group control (control), group early G-CSF (G-CSF 60 min after TE), group rt-PA (rt-PA 60 min after TE), group com (combination rt-PA/G-CSF), group delayed rt-PA (rt-PA after 180 min), group deco (G-CSF after 60 min, rt-PA after 180 min). Animals were investigated by magnetic resonance imaging (MRI) and silver infarct staining (SIS) 24 hours after TE.

Results: Early G-CSF or rt-PA reduced the infarct size compared to all groups ( $p<0.05$ to $p<0.01)$ with the exception of group com, ( $p=$ n.s.) as measured by T2, DWI, and SIS. Late administration of rt-PA lead to high mortality and larger infarcts compared to all other groups ( $p<0.05$ to $p<0.01$ ). Pre-treatment by G-CSF (deco) reduced infarct site compared to delayed rt-PA treatment $(p<0.05)$. G-CSF did not significantly influence PWI when combined with rt-PA. All animals treated by rt-PA showed improved parameters in PWI indicating reperfusion.

Conclusions: G-CSF was neuroprotective when given early after TE. Early combination with rt-PA showed no additional benefit compared to rt-PA or G-CSF alone, but did not lead to side effects. Pretreatment by G-CSF was able to reduce deleterious effects of late rt-PA treatment.
\end{abstract}

Granulocyte Colony-Stimulating Factor (G-CSF) is neuroprotective in models of acute experimental cerebral ischemia [1-9]. During the acute phase of ischemic stroke, various neuroprotective effects of G-CSF have been described in different species [1,2,9]. G-CSF influences apoptotic pathways [3,4], suppresses edema formation and interleukin-1 beta expression $[1,6]$ induces the cerebral G-CSF receptor [7], and diminishes glutamate induced neurotoxicity $[1,10]$. Moreover, reduction of infarct size is associated with an improved functional score $[6,8,11]$. Remarkably, G-CSF reduced the infarct size even when given 72 hours after induction of cerebral ischemia [8]. In addition, G-CSF stimulates endogenous neurogenesis and vascularisation $[6,7,9,11,12]$. As a result, clinical studies are currently conducted to test the

\footnotetext{
*Correspondence: rainer.kollmar@uk-erlangen.de

1 Department of Neurology, University of Erlangen, Erlangen, Germany Full list of author information is available at the end of the article
}

safety and effectiveness of G-CSF after acute ischemic stroke $[9,11,13]$.

Because of its multiple ways of action and good clinical tolerability for other medical conditions [9], G-CSF might be an ideal drug for the treatment of acute ischemic stroke. So far, thrombolysis by recombinant tissue-plasminogen activator (rt-PA) within the first 4.5 hours after symptom onset is the only proven effective therapy for thromboembolic stroke $[14,15]$. As recanalisation and neuroprotection are probably the most promising therapeutical approaches in stroke, combination of rt-PA and G-CSF need to be tested experimentally before using it in patient trials. G-CSF might decrease infarct volume when combined with rt-PA, but may interfere with potentially beneficial effects of rt-PA such as improvement of cerebral blood flow (CBF). Additionally, pretreatment by GCSF might influence infarct volume and overall outcome after delayed and therefore potentially harmful reperfusion. In this context, the interaction of rt-PA associated 
pathways and simultaneous treatment by hematopoetic growth factors is of high clinical relevance, since combination treatment by erythropoetin (EPO) and rt-PA lead to increased side effects in a recently published randomized study [16]. It is very remarkable that no animal experiments have been published for combination of rtPA and EPO before starting the clinical study. Therefore, we investigated whether G-CSF was neuroprotective in a model of thromboembolic stroke. Moreover, the combination therapy with rt-PA was evaluated to eludicate whether G-CSF alters infarct growth in combination therapy. Pretreatment with G-CSF before delayed rt-PA administration was investigated to evaluate whether reperfusion associated injury could be influenced.

\section{Materials and methods}

\section{Animals and experimental groups}

The animal experiments were performed after approval of the animal care committee (Regierungspräsidium Karlsruhe, Germany). Before surgery male Wistar rats (n = 72) weighing 280 to 320 g (Charles-River Deutschland, Sulzfeld, Germany) were assigned to one of the following groups.

- Group control $(n=12)$ : Thromboembolic cerebral ischemia (TE). No specific treatment.

- Group early G-CSF( $n=12)$ : TE followed by intravenous G-CSF treatment after $60 \mathrm{~min}$.

- Group early $\boldsymbol{r t - P A}(n=12)$ : TE followed by intravenous rt-PA treatment $60 \mathrm{~min}$ after TE.

- $\operatorname{Group} \operatorname{com}(n=12)$ : TE followed by intravenous rt-PA treatment $60 \mathrm{~min}$ after TE and intravenous G-CSF treatment 60 min after TE.

- Group delayed $\boldsymbol{r t}-\boldsymbol{P A}(n=12)$ : TE followed by intravenous rt-PA treatment $180 \mathrm{~min}$ after TE.

- $\operatorname{Group} \operatorname{deco}(n=12)$ : TE followed by intravenous GCSF treatment after $60 \mathrm{~min}$ and intravenous rt-PA treatment 180 min after TE.

All animals were subjected to MRI monitoring including perfusion weighted imaging (PWI), diffusion weighted imaging (DWI), T2, and $\mathrm{T} 2 *$ at $0.5,2.5,4$, and 24 hours after TE followed by silver-infarct staining (SIS) as described below.

\section{Animal preparation}

For induction of anesthesia, the animals inhaled a gas mixture of halothane (Forene; Abott, Wiesbaden, Germany), nitrous oxyide (70\%) and oxygen (30\%) via a precalibrated vaporizer (Fortec; Cyprane Keighley, United Kingdom). The right femoral artery and vein were cannulated using polyethylene catheters (PE-50; Labokron, Sinsheim, Germany). Body temperature was kept constant at $37^{\circ} \mathrm{C}$ with a temperature-controlled heating pad (Föhr Medical Instruments, Germany) during surgery. The correlation between body temperature, pericra- nial and intracranial temperature has been shown before [17].

TE was induced as previously described [18]. Briefly, the right common carotid (CCA), internal carotid (ICA), and external carotid artery (ECA) were exposed and further dissection identified the origin of the pterygopalatine artery (PPA). The ECA and the PPA were permanently ligated while the CCA was only temporarily clipped for embolization. A PE 50 catheter was inserted into the ECA proximal to its ligation and 12 red blood clots (each $0.35 \mathrm{~mm}$ in diameter and $3 \mathrm{~mm}$ in length) were injected at the origin of the right middle cerebral artery (MCA). The whole surgical procedure lasted 30 to $40 \mathrm{~min}$.

\section{Rt-PA and G-CSF treatment}

Rt-PA (Alteplase, Boehringer Ingelheim, Ingelheim am Rhein, Germany) was infused intravenously at a dose of $10 \mathrm{mg} / \mathrm{kg}$ body weight (b.w.). Ten percent were given as a bolus at the beginning of thrombolysis followed by continuous infusion over a 30-minute period with a Harvard pump (Harvard Apparatus). The dose was in accordance with experimental studies $[18,19]$. Rt-PA dissolved with 1 $\mathrm{ml}$ of saline $0.9 \%$, while pure saline was administered to the animals in the control group a. Recombinant G-CSF (Neupogen, Amgen, Thousand Oaks) in a dose of $60 \mu \mathrm{g} /$ $\mathrm{kg}$ body weight was dissolved in $1 \mathrm{ml}$ of $0.9 \%$ saline and administered over a period of $30 \mathrm{~min} 60 \mathrm{~min}$ after TE. The dose was in accordance with a previous study [1]

\section{MRI protocol}

The animals were examined in a 2.35-T scanner (Biospec 24/40, BRUKER Medizintechnik, Ettlingen, Germany). An actively shielded gradient coil with an inner diameter of $120 \mathrm{~cm}$ was used. This coil was driven by the standard $150 \mathrm{~V} / 100 \mathrm{~A}$ gradient power supply. In this configuration, $180 \mathrm{mT} / \mathrm{m}$ could be reached in $180 \mathrm{~ms}$. A home-built birdcage resonator with an inner diameter of $40 \mathrm{~mm}$ as RF coil was used. MRI examination started within $30 \mathrm{~min}$ after TE and was repeated at $2.5 \mathrm{~h}, 4 \mathrm{~h}$, and $24 \mathrm{~h}$. The protocol included T2-WI, DWI, T2*, and PWI as described previously [18,20,21]. For PWI, a bolus of $0.5 \mathrm{mmol} / \mathrm{kg}$ bw gadodiamide (Omniscan', Amersham, Braunschweig, Germany) was injected at the scan at $30 \mathrm{~min}, 2.5$ hours, and 4 hours.

An investigator blinded for the treatment groups measured the lesion volumes in T2-weighted and DWIweighted MR images by tracing the area of hyperintense regions. The infarct volume was calculated similar to the method described for SIS staining. The apparent rrCBV was calculated from the PWI data, as previously described [18,20,21]. PWI data was assessed in a region of interest (ROI) with an area of $3 \times 3$ pixels at the level of the lateral basal ganglia, which represents a typical isch- 
emic region after occlusion of the MCA. Values were then calculated in percent of the healthy contralateral hemisphere.

\section{Measurement of infarct volume by the silver infarct staining (SIS) method}

The SIS method was used to measure infarct size $[18,22]$. Following the last MRI investigation after 24 hours, the animals were scarified and brain slices were stained according to the SIS method. A modified version of the semi automated method [23] was used to measure the cerebral infarct volume and calculated as follows:

\section{Functional neurological outcome}

All surviving animal were tested for neurological outcome using the neuroscore according to Menzies [24]:0 = no apparent deficit, 1 = contralateral forelimb flexion; $2=$ decreased grip of contralateral forelimb grip while tail pulled; 3 = spontaneous movement in all directions, contralateral circling only if pulled by tail; $4=$ spontaneous contralateral circling. The testing was performed by a coworker who was blinded for the earlier treatment regimen.

\section{Statistical analysis}

Values of the result section and figures are presented as mean \pm S.D. However, functional outcome is given as median and range. After acquiring all the data, the randomization code was broken. ANOVA and subsequent post hoc Fisher protected least significant difference tests were used. A value of $\mathrm{p}<0.05$ was considered statistically significant. Animals that died before the endpoint of 24 hours were not excluded from MRI, but SIS analysis. The nonparametric Kruskall-Wallis test evaluated the neuroscore with subsequent group comparisons by MannWhitney $U$ test. The Chi square test with Yates correction for small numbers was used to test for differences mortality rate.

\section{Results}

\section{Physiological variables}

There were no significant differences in the physiologic variables (data not shown).

\section{Survival}

Survival rate was $75 \%$ for the control group, the early GCSF group, the early rt-PA group, and the deco group. $83 \%$ of the animals survived in com group. In contrast, most animals died in the delayed rt-PA group which was treated by rt-PA 180 min after TE. Only 5 animals survived 24 hours in this subgroup which corresponds to a survival rate of $41 \%$. Therefore, the survival rate was sig- nificantly lower compared to all other experimental groups $(\mathrm{p}<0.05)$. Animals that died prematurely were assessed for the cause of death and showed all large infarcts in the brain territory supplied by the MCA. Therefore, large infarct was probably responsible for the death.

\section{Functional outcome}

Functional outcome was as follows: control group showed a median of 2 (range 2-3), the early G-CSF group had a median of 2 (range 1-2) and the early G-CSF group a median of 2 (range 1-2). Animals of the early combination group showed a non significant trend towards a better outcome compared to all other groups, since the median Menzies score was 1 (range 1-2). Animals of the group which received delayed rt-PA treatment had a median score of 3 (range 2-3). Pretreatment with G-CSF lead to a median Menzies score of 2 (1-3).

\section{Infarct size calculated from SIS after $\mathbf{2 4}$ hours}

The extent of cerebral infarction was $38 \pm 4 \%$ of the right, ischemic hemisphere in the remaining animals of the delayed rt-PA group, $22 \pm 3 \%$ in the control group, $17 \pm$ $3 \%$ in the early G-CSF group, $14 \pm 3 \%$ in com group, and $11 \pm 3 \%$ in the early thrombolysis group. Therefore, the infarct size in the delayed rt-PA group was larger compared to all others $(\mathrm{p}<0.05)$. Moreover, the infarct size in the control group exceeded the early G-CSF, early rt-PA group, and com group ( $p<0.05)$. Animals of the deco group had larger infarcts compared to early rt-PA, $(\mathrm{p}<$ $0.05)$. There was no difference between the control group and the deco group (Figure 1 and 2).

\section{Perfusion weighted imaging}

Analysis of the relative regional cerebral blood volume (rrCBV) at the level of the basal ganglia showed a decrease to below $50 \%$ of the corresponding nonischemic ROIs 30 min after thromboembolic occlusion (data not

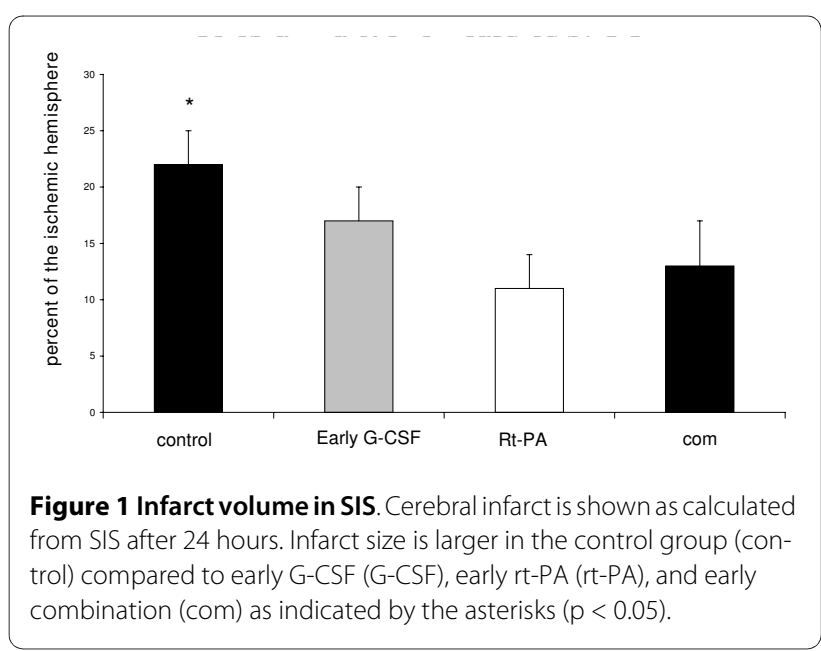




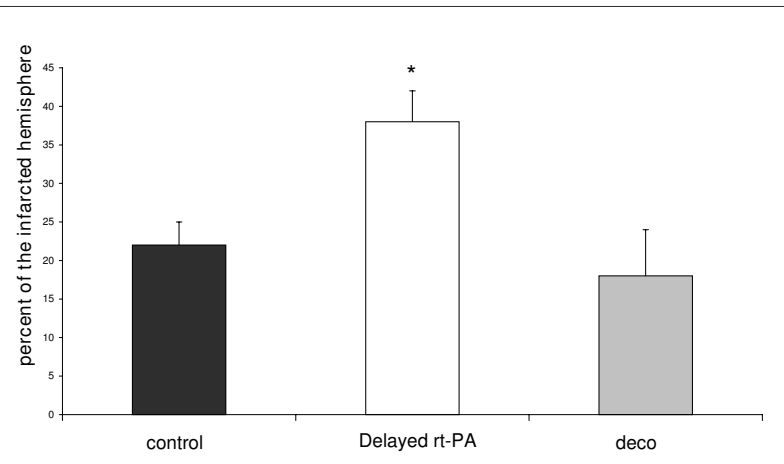

Figure $\mathbf{2}$ Infarct volume in SIS after delayed rt-PA treatment. Cerebral infarct is shown as calculated from SIS after 24 hours. Infarct size is larger in the delayed rt-PA group (delayed rt-PA) compared to the control group (control), and delayed combination (deco) as indicated by the asterisks $(p<0.05)$.

shown). This effect was not different between the groups $(\mathrm{p}=\mathrm{ns})$. The rrCBV slightly was significantly larger in the rt-PA group and com group compared to all other groups, $(\mathrm{p}<0.001)$. There were no significant differences in the groups not treated by rt-PA $(\mathrm{p}=\mathrm{ns}) .4$ hours after stroke onset, rrCBV was larger in the rt-PA group, delayed rt-PA group and the com group compared to the control group and the early G-CSF group, $(\mathrm{p}<0.005)$. There was a significant difference between the rt-PA group $(94 \pm 11 \%)$, delayed rt-PA group $(91 \pm 7 \%)$ compared to the deco group (75 $\pm 4 \%$; $<0.05)$. Data is shown in Table 1 .

\section{Diffusion weighted imaging}

There were no significant differences between the lesion size after 0.5 hours as calculated from DWI ( $\mathrm{p}=\mathrm{ns}$; data not shown).

DWI lesions after 2.5 hours accounted to $43 \pm 5 \mathrm{~mm}^{3}$ in the control group and were significantly larger than those observed for the rt-PA group by $26 \pm 7 \mathrm{~mm}^{3}$, and early com group by $21 \pm 8 \mathrm{~mm}^{3}(\mathrm{p}<0.05)$, (Data shown in Figure 3). There was no significant difference compared to the early G-CSF group by $31 \pm 5 \mathrm{~mm}^{3}$, the deco group by $33 \pm 5 \mathrm{~mm}^{3}$, and the delayed rt-PA group with $42.7 \pm 4$ $\mathrm{mm}^{3}$. Animals treated with early G-CSF tended towards smaller lesions, since the lesion volume was $26 \pm 6 \mathrm{~mm}^{3}$ in the early G-CSF group and $21 \pm 8 \mathrm{~mm}^{3}$ in the com group. Early thormbolysis lead to a lesion volume of 25.9 $\pm 9 \mathrm{~mm}^{3}(\mathrm{p}<0.05) .4$ hours after stroke onset, lesion volume was larger in the delayed rt-PA group $(135.8 \pm 22$ $\left.\mathrm{mm}^{3}\right)$ than in the deco group $\left(80.2 \pm 14 \mathrm{~mm}^{3} ; \mathrm{p}<0.05\right)$, the early G-CSF group $\left(65.1 \pm 17 \mathrm{~mm}^{3} ; \mathrm{p}<0.005\right)$, and early rt-PA (57 $\left.\pm 17 \mathrm{~mm}^{3} ; \mathrm{p}<0.005\right)$. After 24 hours, the lesion volume of the delayed rt-PA group was $180 \pm 13$ $\mathrm{mm}^{3}$ and significantly larger than in the control group (102.4 $\left.\pm 11 \mathrm{~mm}^{3} ; \mathrm{p}>0.001\right)$, the early G-CSF group (71.1 $\left.\pm 8 \mathrm{~mm}^{3} ; \mathrm{p}<0.001\right)$, the deco group $\left(87.3 \pm 12 \mathrm{~mm}^{3} ; \mathrm{p}>\right.$
$0.001)$, and the early rt-PA group $\left(68.3 \pm 9 \mathrm{~mm}^{3} ; \mathrm{p}>\right.$ 0.001 ). Moreover, lesion volume in the early G-CSF group and the early rt-PA group was smaller than in the control group $(\mathrm{p}<0.05)$.

Values of DWI after 24 hours show significant differences between the treatment groups (Table 1 and 2). The infarct volume was larger in the control group a vs. early G-CSF group ( $\mathrm{p}=0.019)$, to early rt-PA group $(\mathrm{p}=0.006)$, and the com group $(\mathrm{p}=0.013)$. No treatment resulted in smaller infarct volume than G-CSF plus delayed rt-PA administration in group deco $(\mathrm{p}=0.002)$. There was a non-significant trend towards smaller infarct volume in group deco compared to the control group $(\mathrm{p}=0.25$; n.s.). Comparing the early treatment group G-CSF and rt-PA, there was no difference for the infarct volume. Combining rt-Pa and G-CSF (group com) in the early phase lead to smaller infarct than for rt-PA treatment alone $(\mathrm{p}=$ 0.001 ). Early rt-PA treatment (group rt-PA) and combination (group com) lead to smaller infarcts than in group delayed rt-PA $(\mathrm{p}=0.001)$. Pretreatment by G-CSF in combination with delayed rt-PA in group deco reduced the infarct volume compared to the delayed rt-PA group $(\mathrm{p}=0.001)$.

\section{T2-WI and T2*-WI}

There were no significant differences among all groups at the first scan after $30 \mathrm{~min}$ (data not shown) and after 2.5 hours $(\mathrm{p}=\mathrm{ns})$. After 4 hours, lesion size in the delayed rtPA group was $129.4 \pm 14.4 \mathrm{~mm}^{3}$ and therefore larger than in the early G-CSF group $\left(65.3 \pm 18 \mathrm{~mm}^{3}\right)$, the com group $\left(68.1 \pm 13 \mathrm{~mm}^{3}\right)$, and the rt-PA group $\left(58.1 \pm 12 \mathrm{~mm}^{3}\right)$; $(\mathrm{p}$ $<0.005)$. After 24 hours, infarct size was larger in the control $\left(113 \pm 7 \mathrm{~mm}^{3}\right)$ compared to the early G-CSF group $\left(85.5 \pm 8 \mathrm{~mm}^{3} ; \mathrm{p}<0.05\right)$, the com group $\left(70.3 \pm 15 \mathrm{~mm}^{3} ; \mathrm{p}\right.$ $<0.01)$, and the early rt-PA group $\left(59.1 \pm 8 \mathrm{~mm}^{3}\right) ;(\mathrm{p}<$ $0.005)$. However, infarct size in the control group was smaller compared to the delayed rt-AP group (193.3 \pm 5 $\left.\mathrm{mm}^{3} ; \mathrm{p}<0.001\right)$. Moreover, the lesion size in the delayed rt-PA group was larger than in the com group, the early G-CSF group, and early rt-PA group ( $<0.001)$. T2*-WI showed 4 animals (one in each group excluding early thrombolysis) with an intra-cerebral hemorrhage.

\section{Discussion}

This study shows for the first time that G-CSF reduces infarct volume in a model of thromboembolic stroke. Moreover, it prevents some deleterious effects of delayed rt-PA treatment in terms of infarct growth and mortality during an observation time of 24 hours after stroke onset. These results are an important step towards further clinical investigations on G-CSF and acute stroke, since the EPO trial failed because of deleterious combination therapy of EPO and rt-PA [16]. 
Table 1: Data of serial MRI.

\section{DWI in $\mathbf{m m} 3$}

\begin{tabular}{|c|c|c|c|}
\hline & $2.5 \mathrm{hrs}$ & $4 \mathrm{hrs}$ & $24 \mathrm{hrs}$ \\
\hline control & $43 \pm 5$ & $86 \pm 14$ & $102 \pm 11$ \\
\hline early G-CSF & $31 \pm 5$ & $65 \pm 17$ & $71 \pm 8$ \\
\hline rt-PA & $26 \pm 7$ & $57 \pm 17$ & $68 \pm 9$ \\
\hline com & $21 \pm 8$ & $49 \pm 14$ & $65 \pm 11$ \\
\hline delayed rt-PA & $43 \pm 4$ & $136 \pm 22$ & $180 \pm 13$ \\
\hline deco & $33 \pm 5$ & $80 \pm 14$ & $87 \pm 12$ \\
\hline
\end{tabular}

T2 in $\mathbf{m m 3}$

\begin{tabular}{|c|c|c|}
\hline & $4 \mathrm{hrs}$ & 24 hrs \\
\hline control & $79 \pm 12$ & $113 \pm 7$ \\
\hline early G-CSF & $65 \pm 18$ & $85 \pm 8$ \\
\hline rt-PA & $68 \pm 13$ & $69 \pm 8$ \\
\hline com & $54 \pm 16$ & $70 \pm 15$ \\
\hline delayed rt-PA & $129 \pm 14$ & $193 \pm 15$ \\
\hline \multirow[t]{3}{*}{ deco } & $68 \pm 13$ & $93 \pm 13$ \\
\hline & & PWI in \% \\
\hline & $2.5 \mathrm{hrs}$ & $4 \mathrm{hrs}$ \\
\hline control & $28 \pm 3$ & $58 \pm 7$ \\
\hline early G-CSF & $34 \pm 5$ & $53 \pm 8$ \\
\hline rt-PA & $78 \pm 4$ & $94 \pm 11$ \\
\hline com & $75 \pm 6$ & $91 \pm 7$ \\
\hline
\end{tabular}


Table 1: Data of serial MRI. (Continued)

\begin{tabular}{ccc}
\hline delayed rt-PA & $34 \pm 3$ & $90 \pm 5$ \\
\hline deco & $38 \pm 7$ & $75 \pm 4$ \\
\hline
\end{tabular}

These are given as means \pm SD for different time points after stroke onset.

\section{Neuroprotective effects of G-CSF}

Recent experimental studies showed that G-CSF is beneficial after cerebral ischemia and brain injury [1-8]. While neuroprotective effects are described in the early stage of brain injury, G-CSF also stimulates neuronal progenitor cells providing a link to functional recovery $[6,9]$. G-CSF reduces infarct volume after transient suture occlusion of the MCA and protects neurons against glutamateinduced excitotoxicity in cell culture $[1,10]$. Further neuroprotective mechanisms include an increased STAT3 regulation in the penumbra of G-CSF-treated rats. Effects of G-CSF are probably mediated by a special neuronal GCSF receptor [1,7], since G-CSF passes even the intact the blood-brain-barrier [7] and therefore reaches injured brain regions. Moreover, G-CSF seems to have additional regenerative effects as bone marrow cells are activated. Neuronal plasticity and vascularisation were proven in experimental studies of cerebral ischemia [6,7].

\section{G-CSF compared to early thrombolysis}

So far, G-CSF was not tested in models of thromboembolic stroke. However, this step is essential when transfer-

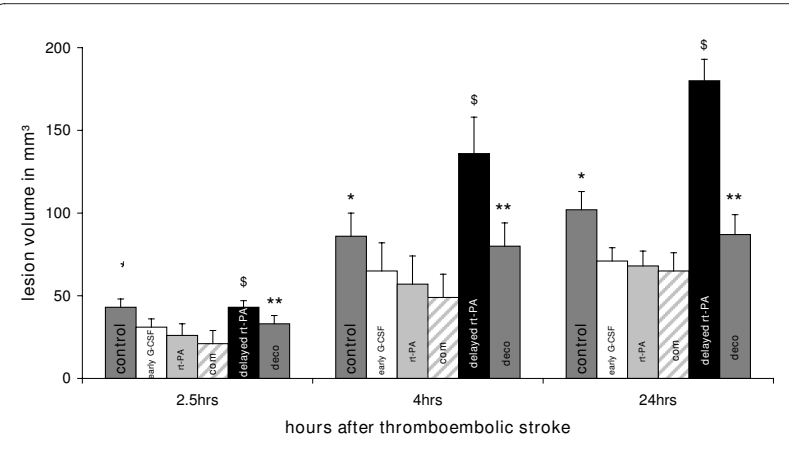

Figure $\mathbf{3}$ Lesion volume in MRI. Lesion volume is shown as calculated from DWI and expressed as means \pm SD. The lesion volume in the control group was larger compared to early rt-PA (rt-PA) and early combination group (com) after 2.5 hours, and larger than the early rt-PA, early G-CSF (G-CSF) and early combination group after 4 and 24 hours as shown by the single asterisks. Delayed rt-PA treatment (delayed rt$P A$ ) resulted in larger lesion volume than the early rt-PA, early G-CSF and early combination group after 2.5 hours, and additionally larger than the control group after 4 hours. It was larger than all others after 24 hours as shown by the string sign. The late combination group (deco) showed larger infarcts than the early combination after 2.5 hours. After 4 hours, lesion volume was larger than all others with exception of delayed rt-PA. After 24 hours, there was a difference compared to the early rt-PA and early combination as indicated by the double asterisks. Levels of significance are given in table 2 . ring neuroprotective agents to stroke patients. If neuroprotectants are successful at all, the chance is the highest in early stages of stroke. In this study, MRI-data as well as SIS showed that G-CSF was as effective as early thrombolysis in terms of reduction of infarct volume. There was no difference in mortality as well. G-CSF did not influence rrCBV compared to the control group. As expected, early rt-PA treatment resulted in almost complete normalisation of rCBV. It can be suggested that the mentioned multiple neuroprotective effects contribute to reduction of infarct for this subgroup. These results are in accordance to former studies in suture occlusion model $[1,6]$.

\section{G-CSF prior to delayed thrombolysis}

G-CSF reduced infarct volume and mortality when given prior to delayed rt-PA treatment. Moreover, there was no significant difference to early rt-PA and G-CSF treatment alone. In accordance to data of the suture occlusion model, delayed restoration of CBF leads to larger infarct than early restoration. Aronowski et al. observed larger infarct volumes and edema as compared to permanent ischemia, when the MCA occluding suture was removed 120 to $300 \mathrm{~min}$ after induction of ischemia [25]. Clinical data shows that delayed thrombolysis beyond the three hours window in already demarked infarct increases the risk of side effects such as hemorrhage or enlargement of infarction [14]. Probably these side effects are caused by reperfusion-associated injury and neurotoxic properties of rt-PA [26,27]. In contrast to the patient, infarct mature much faster in rats and therefore a time window of three hours can be considered as late thrombolysis. In accordance, early rt-PA treatment lead to smaller infarcts compared to the control group, while delayed administration of rt-PA increased infarct volume and mortality in this study. It can be suggested that G-CSF helps to prolong the time window for thrombolysis. This is of importance, since the transport of patients to the clinic is often long and excludes them from thrombolytic treatment. Pretreatment In the ambulance car or if the treatment with rt-PA is delayed in the hospital might be helpful for these subgroup of stroke patients.

However, there are several limitations of the study. Certainly, observation periods of more than 24 hours are necessary to test whether effects of neuroprotectants are transient or permanent. Moreover, further investigations should address whether G-CSF interacts with rt-PA and 


\section{Table 2: Comparing diffusion-weighted imaging}

\begin{tabular}{|c|c|c|c|c|c|c|}
\hline $\begin{array}{c}\text { after } 2.5 \\
\text { hours }\end{array}$ & & & & & & \\
\hline & control & $\begin{array}{c}\text { early } \\
\text { G-CSF }\end{array}$ & rt-PA & com & delayed rt-PA & deco \\
\hline control & & n.s. & $<0.05$ & $<0.05$ & n.s. & n.s. \\
\hline early G-CSF & n.s. & & n.s. & n.s. & $<0.05$ & n.s. \\
\hline rt-PA & $<0.05$ & n.s. & & n.s. & $<0.05$ & n.s. \\
\hline com & n.s. & n.s. & n.s. & & $<0.05$ & n.s. \\
\hline delayed rt-PA & n.s. & $<0.05$ & $<0.05$ & $<0.05$ & & n.s. \\
\hline deco & n.s. & n.s. & n.s. & $<0.05$ & n.s. & \\
\hline \multicolumn{7}{|l|}{ after 4 hours } \\
\hline & control & $\begin{array}{c}\text { early } \\
\text { G-CSF }\end{array}$ & rt-PA & com & delayed rt-PA & deco \\
\hline control & & n.s. & $p<0.05$ & $p<0.01$ & $<0.05$ & n.s. \\
\hline early G-CSF & n.s. & & n.s. & n.s. & $<0.005$ & n.s. \\
\hline rt-PA & $p<0.05$ & n.s. & & n.s. & $<0.005$ & n.s. \\
\hline com & $p<0.01$ & n.s. & n.s. & & $<0.005$ & n.s. \\
\hline delayed rt-PA & $<0.05$ & $<0.005$ & $<0.005$ & $<0.005$ & & $<0.05$ \\
\hline deco & n.s. & n.s. & n.s. & n.s. & $<0.05$ & \\
\hline \multicolumn{7}{|l|}{$\begin{array}{c}\text { after } 24 \\
\text { hours }\end{array}$} \\
\hline & control & $\begin{array}{c}\text { early } \\
\text { G-CSF }\end{array}$ & rt-PA & com & delayed rt-PA & deco \\
\hline control & & $<0.05$ & $<0.05$ & $<0.05$ & $<0.001$ & n.s. \\
\hline early G-CSF & $<0.05$ & & n.s. & n.s. & $<0.001$ & n.s. \\
\hline
\end{tabular}


Table 2: Comparing diffusion-weighted imaging (Continued)

\begin{tabular}{ccccc}
\hline rt-PA & $<0.05$ & n.s. & n.s. & $<0.001$ \\
\cline { 1 - 3 } com & $<0.05$ & n.s. & $<0.001$ & $<0.05$ \\
\hline delayed rt-PA & $<0.001$ & $<0.001$ & $<0.001$ & $<0.001$ \\
\hline deco & n.s. & n.s. & $<0.05$ & $<0.05$ \\
\hline
\end{tabular}

Levels of significant difference are given here as calculated from DWI-sequences.

CBF. Autoradiographic techniques may answer these questions, but were not in the focus of the present study. Animals were exposed to anesthesia for several hours. While this could interfere with mortality overall, differences between the groups cannot explained with it. Although we did not investigate further pathways of GCSF and combination with rt-PA, this study is essential when G-CSF will be investigated in patients treated by rtPA.

In conclusion, the results of the present study are encouraging on the path of new therapies for ischemic stroke. G-CSF represents an interesting and promising candidate for stroke therapy because of its neuroprotective properties, potential induction of stem cells and good clinical tolerance in hematological patients. Further experimental studies have to investigate combination therapy of G-CSF and rt-PA over longer time periods, since combining the so far best medical therapy rt-PA with new drugs represents a logical and potentially successful way for stroke treatment.

\section{Competing interests}

The authors RK and SS are involved into the AXIS-trial investigating safety and feasibility of G-CSF after acute stroke. Moreover, they own a patent on the use of growth-factors such as G-CSF for the treatment of stroke.

\section{Authors' contributions}

RK and SS designed the study, did the statistics and prepared the manuscript. $\mathrm{NH}$ and $\mathrm{CU}$ performed the experiments. $\mathrm{NH}$ investigated neurological examination of the animals. All authors read and approved the final manuscript.

\section{Author Details}

'Department of Neurology, University of Erlangen, Erlangen, Germany, 2Department of Neurology, University of Massachusetts Medical School, Worcester, USA and ${ }^{3}$ Neurology Hospital, Ludwigshafen, Ludwigshafen, Germany

Received: 18 February 2010 Accepted: 14 April 2010

Published: 14 April 2010

\section{References}

1. Schabitz WR, Kollmar R, Schwaninger M, Juettler E, Bardutzky J, Scholzke MN, Sommer C, Schwab S: Neuroprotective effect of granulocyte colony-stimulating factor after focal cerebral ischemia. Stroke 2003, 34:745-51.

2. Six I, Gasan G, Mura E, Bordet R: Beneficial effect of pharmacological mobilization of bone marrow in experimental cerebral ischemia. Eur J Pharmacol 2003, 458:327-8.
3. Komine-Kobayashi M, Zhang N, Liu M, Tanaka R, Hara H, Osaka A, Mochizuki H, Mizuno Y, Urabe T: Neuroprotective effect of recombinant human granulocyte colony-stimulating factor in transient focal ischemia of mice. J Cereb Blood Flow Metab 2006, 26:402-13.

4. Solaroglu I, Tsubokawa T, Cahill J, Zhang JH: Anti-apoptotic effect of granulocyte-colony stimulating factor after focal cerebral ischemia in the rat. Neuroscience 2006, 143:965-74

5. Gibson CL, Bath PM, Murphy SP: G-CSF reduces infarct volume and improves functional outcome after transient focal cerebral ischemia in mice. J Cereb Blood Flow Metab 2005, 25:431-9.

6. Shyu WC, Lin SZ, Yang HI, Tzeng YS, Pang CY, Yen PS, Li H: Functional recovery of stroke rats induced by granulocyte colony-stimulating factor-stimulated stem cells. Circulation 2004, 110:1847-54.

7. Schneider A, Kruger C, Steigleder T, Weber D, Pitzer C, Laage R, Aronowski J, Maurer MH, Gassler N, Mier W, Hasselblatt M, Kollmar R, Schwab S, Sommer C, Bach A, Kuhn HG, Schabitz WR: The hematopoietic factor GCSF is a neuronal ligand that counteracts programmed cell death and drives neurogenesis. J Clin Invest 2005, 115:2083-98.

8. Schneider A, Wysocki R, Pitzer C, Kruger C, Laage R, Schwab S, Bach A Schabitz WR: An extended window of opportunity for G-CSF treatment in cerebral ischemia. BMC Biol 2006, 18(4):36.

9. Schneider A, Kuhn HG, Schabitz WR: A role for G-CSF (granulocytecolony stimulating factor) in the central nervous system. Cell Cycle 2005, 4:1753-7.

10. Han JL, Blank T, Schwab S, Kollmar R: Inhibited glutamate release by granulocyte-colony stimulating factor after experimental stroke. Neurosci Lett 2008, 432:167-9.

11. Shyu WC, Lin SZ, Lee CC, Liu DD, Li H: Granulocyte colony-stimulating factor for acute ischemic stroke: a randomizedcontrolled trial. CMAJ 2006, 174:927-33.

12. Kawada H, Takizawa S, Takanashi T, Morita Y, Fujita J, Fukuda K, Takagi S, Okano H, Ando K, Hotta T: Administration of hematopoietic cytokines in the subacute phase after cerebral infarction is effective for functional recovery facilitating proliferation of intrinsic neural stem/progenitor cells and transition of bone marrow-derived neuronal cells. Circulation 2006, 113:701-10

13. Sprigg N, Bath PM, Zhao L, Willmot MR, Gray LJ, Walker MF, Dennis MS, Russell N: Granulocyte-colony-stimulating factor mobilizes bone marrow stem cells in patients with subacute ischemic stroke: the Stem cell Trial of recovery EnhanceMent after Stroke (STEMS) pilot randomized, controlled trial (ISRCTN 16784092). Stroke 2006, 37:2979-83

14. Hacke W, Donnan G, Fieschi C, Kaste M, von Kummer R, Broderick JP, Brott T, Frankel M, Grotta JC, Haley EC Jr, Kwiatkowski T, Levine SR, Lewandowski C, Lu M, Lyden P, Marler JR, Patel S, Tilley BC, Albers G, Bluhmki E, Wilhelm M, Hamilton S: Association of outcome with early stroke treatment: pooled analysis of ATLANTIS, ECASS, and NINDS rt-PA stroke trials. Lancet 2004, 363:768-774.

15. Hacke W, Kaste M, Bluhmki E, Brozman M, Dávalos A, Guidetti D, Larrue V, Lees KR, Medeghri Z, Machnig T, Schneider D, von Kummer R, Wahlgren N, Toni D, ECASS Investigators: Thrombolysis with alteplase 3 to 4.5 hours after acute ischemic stroke. NEngl J Med 2008, 359:1317-29.

16. Ehrenreich $\mathrm{H}$, Weissenborn $\mathrm{K}$, Prange $\mathrm{H}$, Schneider D, Weimar $\mathrm{C}$, Wartenberg K, Schellinger PD, Bohn M, Becker H, Wegrzyn M, Jähnig P, Herrmann M, Knauth M, Bähr M, Heide W, Wagner A, Schwab S, Reichmann H, Schwendemann G, Dengler R, Kastrup A, Bartels C, EPO 
Stroke Trial Group: Recombinant human erythropoietin in the treatment of acute ischemic stroke. Stroke 2009, 40:e647-56.

17. Frietsch T, Krafft P, Piepgras A, Lenz C, Kuschinsky W, Waschke KF: Relationship between local cerebral blood flow and metabolism during mild and moderate hypothermia in rats. Anesthesiology 2000, 92:754-63.

18. Kollmar R, Henninger N, Bardutzky J, Schellinger PD, Schabitz WR, Schwab $\mathrm{S}$ : Combination therapy of moderate hypothermia and thrombolysis in experimental thromboembolic stroke - an MRI study. Exp Neurol 2004 190:204-212.

19. Korninger C, Collen D: Studies on specific fibrinolytic effect of human extrinsic plasminogen activator in human blood and in various animal species in vitro. Thromb Haemost 1981, 46:561-565.

20. Heiland S, Benner T, Reith W: Perfusion-weighted MRI using gadobutrol as a contrast agent in a rat stroke model. J Magn Reson Imaging 1997, 7:1109-1115.

21. Heiland S, Sartor K: Magnetic resonance tomography in stroke: its methodological bases and clinical use [in German]. Rofo Fortschr Geb Rontgenstr Neuen Bildgeb Verfahr 1999, 171:3-14

22. Vogel J, Mobius C, Kuschinsky W: Early delineation of ischemic tissue in rat brain cryosections by high-contrast staining. Stroke 1999, 30:1134-1141.

23. Swanson RA, Morton MT, Tsao-Wu G, Savalos RA, Davidson C, Sharp FR: A semiautomated method for measuring brain infarct volume. J Cereb Blood Flow Meta 1990, 10:290-293.

24. Menzies SA, Hoff JT, Betz AL: Middle cerebral artery occlusion in rats: A neurological and pathological evaluation of a reproducible model. Neurosurgery 1992, 31:100-106.

25. Aronowski J, Strong R, Grotta JC: Reperfusion injury: Demonstration of brain damage produced by reperfusion after transient focal ischemia in rats. J Cereb Blood Flow Metab 1997, 17:1048-1056.

26. Asahi M, Asahi K, Wang X, Lo EH: Reduction of tissue plasminogen activator-induced hemorrhage and brain injury by free radical spin trapping after embolic focal cerebral ischemia in rats. $J$ Cereb Blood Flow Metab 2000, 20:452-457.

27. Wang X, Asahi M, Lo EH: Tissue type plasminogen activator amplifies hemoglobin-induced neurotoxicity in rat neuronal cultures. Neurosci Lett 1999, 274:79-82.

doi: 10.1186/2040-7378-2-9

Cite this article as: Kollmar et al., G-CSF, rt-PA and combination therapy after experimental thromboembolic stroke Experimental \& Translational Stroke Medicine 2010, 2:9

\section{Submit your next manuscript to BioMed Central} and take full advantage of:

- Convenient online submission

- Thorough peer review

- No space constraints or color figure charges

- Immediate publication on acceptance

- Inclusion in PubMed, CAS, Scopus and Google Scholar

- Research which is freely available for redistribution

Submit your manuscript at www.biomedcentral.com/submit
C Biomed Central 